\title{
COVID-19 threatens maternal mental health and infant development: possible paths from stress and isolation to adverse outcomes and a call for research and practice
}

\author{
Amanda Venta ${ }^{1}$ D . Johanna Bick ${ }^{1} \cdot$ Jeremy Bechelli ${ }^{2}$ \\ Accepted: 5 February 2021 / Published online: 22 February 2021 \\ (c) The Author(s), under exclusive licence to Springer Science+Business Media, LLC part of Springer Nature 2021
}

\begin{abstract}
The COVID-19 pandemic exposed mothers to stress and social isolation during the pre- and post-natal periods. The deleterious effects of stress on both pregnant women and their infants are well documented, with research suggesting that effects are exacerbated by reduced social support. In this brief report, we summarize evidence linking stress and social isolation to negative outcomes for mothers and infants and present a conceptual model featuring inflammation as a driving mechanism. There is strong evidence that the coronavirus pandemic will affect mothers and infants through immune pathways that, in previous research, have been shown to link stress and social isolation during the pre- and post-natal periods with deficits in maternal mental health and infant well-being and development across developmental stages. We close with recommendations for novel research, policy changes, and integrated clinical care that can address these biological threats to infants and mothers while leveraging the anti-inflammatory effects of social support.
\end{abstract}

Keywords COVID-19 $\cdot$ Pregnancy $\cdot$ Inflammation $\cdot$ Mental health $\cdot$ Attachment

\section{Background and Preliminary Evidence on the Topic}

The coronavirus (COVID-19) pandemic has produced highly stressful situations for many families due to health anxiety, grief, extreme social distancing, and financial insecurity. Across the globe, the number of infants born during shelterin-place orders alone likely exceeded 31 million, and, certainly, infants have continued to be born in unusual circumstances since shelter-in-place orders were lifted. Stress and social isolation during the perinatal and postpartum periods are associated with adverse outcomes for mothers and infants, threatening the family system and putting an enormous number of families at risk right now. We face a critical need for rapid research and novel clinical interventions.

Amanda Venta

aventa@uh.edu

1 Department of Psychology, University of Houston, 4849 Calhoun Rd., Room 373, Houston, TX 77204-6022, USA

2 Department of Biological Sciences, Sam Houston State University, Huntsville, TX, USA
Social support is strongly related to child development across multiple levels of analysis. Indeed, mother perceived social support during infant and toddler stages is associated with reduced parenting stress [1], increased maternal responsiveness and stimulation towards infants [2], more positive mother-child interactions [3], reduced maternal depression [4], and reduced maternal anxiety and harsh behavior [5]. Likewise, there are direct relations of maternal perceived social support with reduced childhood cognitive abilities [6] as well as with increased child behavior problems, depression, and accident proneness with effects stretching into adolescence [5]. Much of this research posits that isolation increases maternal stress which has broad, toxic effects. Indeed, there are long term adverse outcomes for children when mothers are exposed to even a temporary stressor like an ice storm [7] or another natural disaster [8]. These studies reveal increased risk for adverse developmental outcomes in cognitive, behavioral, motor, and physical domains [9].

Maternal stress and social isolation get under the skin of both mothers and babies [10], explaining their broad, negative effects. The inflammatory hypothesis of neurodevelopment [11] explains that proinflammatory mediators indirectly link perinatal and postpartum maternal stress with brain and behavioral development of infants, with 
crosscutting neurodevelopmental consequences. This process is perpetuated throughout infancy through breastfeeding, which passes cytokines from mothers to infants. The effects of early inflammation on development are clear: within the first month of life, elevated inflammation in pre-term infants can be detected and linked prospectively to adverse outcomes at ages 2 and 10 [12-14]. Two studies have replicated these findings in infants living in poverty, linking inflammation in infants to development at 1-2 and 7-9 years, independent of income, and documenting protective effects for anti-inflammatory cytokines [11, 15]. Findings suggest that cytokines act as a common mediator, explaining the effects of maternal adversity on babies both prenatally and during infancy on development, and that anti-inflammatory cytokines protect children from demonstrating negative developmental consequences in the context of adversity. We can only assume that the stress of being pregnant and giving birth during a global pandemic - particularly when coupled with necessary social isolation and possibly other sources of stress including financial strain, job loss, grief, and illness - is wreaking havoc on the immune systems of mothers and babies, disrupting many aspects of their health and family systems.

\section{Implications for Research and Practice}

Research on the psychological and biological cascades of stress and social isolation on mothers and infants is needed immediately. We must move quickly to understand the risk of long-term adversity for these families and, relatedly, identify protective factors that can be leveraged to mitigate the catastrophe of adverse outcomes for this birth cohort. Identification of the physiological process-inflammation response-by which maternal stress and isolation following an impactful event translate to persistent adverse outcomes in children's neurodevelopmental, cognitive, and psychosocial outcomes has significant therapeutic implications. To date there has been little research on the possible protective effects of anti-inflammatory cytokines in infants and no research on its potential therapeutic value despite therapeutic use in other domains of healthcare (e.g., [16]).

Further, psychological research could motivate changes in gynecological and pediatric standard of care. Indeed, research could motivate making maternal stress screening a standard component of prenatal and postpartum care, with relevant intervention studies in the future targeting this risk factor. Likewise, health care policies could build in support calls with mothers, particularly in contexts such as social-distancing or for mothers who will lack social support for other reasons, recognizing that social support reduces inflammation in other medical groups $[17,18]$. If contextual risks (like stress and isolation) cannot feasibly be mitigated, intervening to foster secure attachment may have similar protective effects given the anti-inflammatory effects of secure attachment relationships [19, 20]. While there are many attachment interventions [21], they are not currently being used to buffer the long term cascade of negative outcomes associated with perinatal and postpartum stress and isolation. As such, these treatments are not being used to mitigate biological risk for adverse child outcomes among mother-infant dyads exposed to contextual risk (like social isolation), despite extensive work making these treatments acceptable and feasible (e.g., home visit format) for use with families from diverse ethnic, racial, and socioeconomic backgrounds.

The number of children born under conditions of stress and social isolation stretches far beyond this pandemic. Maternal stress and social isolation are common among single mothers and families living in poverty. Biological and behavioral cascades of social isolation observed the during COVID-19 pandemic could help develop interventions to target risk mechanisms (and capitalize upon protective mechanisms) in these other vulnerable groups. Indeed, inflammatory mechanisms have broad public health impact: chronically elevated levels of inflammatory cytokines have been linked with serious medical complications [22] and psychological and developmental consequences, including Autism [9].

\section{Recommendations}

\section{Capitalize Upon the COVID-19 Pandemic to Conduct New Empirical Research}

The aforementioned studies of maternal social support, stress, and infant well-being are observational; social isolation and stress are outside of the realm of ethical experimental manipulation. Though extant research has linked reduced social support and increased stress to adverse outcomes for mothers and children, these variables are confounded with others like a mother's temperament or psychopathology, financial resources, and marital satisfaction. In contrast, the COVID-19 pandemic provides a natural experiment in which pregnant women, regardless of random variation in the aforementioned confounds and other unknowns, have been forced into a period of stress and social isolation, allowing the effects of stress and isolation to be measured and disentangled from other contextual risk factors, as has been done in the aftermath of natural disasters before [8,9]. Several specific areas for future research are recommended:

1. Assess infant developmental and maternal mental health outcomes during COVID-19 and in the aftermath. A systematic review of child and parent outcomes in the 
context of previous pandemics revealed that we should expect COVID-19 to produce serious mental health symptoms in caregivers and children (with long term consequences for child development and health) related to fear of infection, adverse childhood experiences, and increased stress [23]. Examining short and long term outcomes for infants born during the COVID-19 pandemic (and their caregivers) will add to this literature base. Recognizing that studies of normal and abnormal development inform one another, comparing children born during the pandemic to those born before and after can teach the scientific community as much about resilient development as about the adverse outcomes that may emerge in relation to stress, social isolation, and other COVID-19-related factors (e.g., infection, material hardship). Specific research questions in need of examination include: How are children born during the COVID-19 different, across developmental domains, from age-, sex-, and socioeconomic-status-matched controls born before and after over time? How did mothers who gave birth during the COVID-19 pandemic experience pregnancy and post-partum adaptation differently from their demographically-matched peers who gave birth under different circumstances? Did some groups of mothers and children (e.g., families from ethnic or racial minority groups) display particularly poor or positive outcomes in comparison to their peers? Parsing the effects of COVID-19 infection versus the effects of COVID-19-related changes in the perinatal environment is a critical need.

2. Examine mechanisms of resilience and risk. While identifying infant and mother outcomes following COVID19 is important in and of itself, future research should endeavor to examine how stress, social isolation, and other COVID-19-related factors translate to risk and resilience for families. In this manuscript, we posit one mechanism, inflammation, by which stress and social isolation might translate to adverse outcomes. However, there are many other behavioral dimensions and neural systems to consider. Specifically: Do behavioral dimensions in the infant, mother, dyad, or broader environment explain COVID-19-related outcomes? Do neural systems in the infant, mother, or perinatal environment explain COVID-19-related outcomes? Identifying the behavioral and biological mechanisms that explain risk is necessary for driving prevention and intervention efforts not just for families affected by COVID-19 but also for families who experience vulnerabilities that mirror those conferred by COVID-19, like mothers who experience social isolation perinatally due to financial hardships, domestic violence, or mental health problems. Likewise, identifying mechanisms that underlie resilience is essential in recruiting protective factors for utilization in interventions and for developing new therapeutics (e.g., anti-inflammatory treatments) that may mitigate adverse outcomes.

3. Pilot interventions for immediate use. Empirical research, particularly intervention development, is slow. If the typical stages of intervention development or adaptation are undertaken prior to making efforts to support infants born during the COVID-19 pandemic and their mothers, we will be too late, missing the most plastic period of child development and one of the most vulnerable periods of a mother's life. For this reason, researchers studying interventions that are unlikely to pose harm to the child and mother may wish to move to intervention pilot testing more rapidly than they would in other circumstances. Early data on caregiver and child outcomes during the COVID-19 pandemic reveal that higher levels of physical exercise [24] and social support [25], for instance, buffer the effects of COVID19-related hardship on maternal and child mental health. While these studies were observational, they suggest that pilot research with behavioral interventions should be prioritized.

\section{Attend to the Experience of Individuals from Racial/ Ethnic Minority Groups}

COVID-19 infections, and perhaps the stress and social isolation related to infection rates, have affected individuals from racial and ethnic minority communities disproportionately. Healthcare systems across the board must recognize that in addition to the deleterious effects of minority stress on birth outcomes [26], women from ethnic minority groups demonstrate particularly pronounced immune responses to stress in pregnancy [27] making them particularly susceptible to the possible consequences of stress and social isolation. Efforts to mitigate stress and social isolation in pregnant women and new mothers from ethnic minority groups should be at the forefront of policy discussions, financial stimulus negations, and healthcare initiatives across obstetrics, pediatrics, and primary care.

\section{Integrate Care to Protect Families}

Though empirical research is needed, it should not delay the immediate roll-out of interventions to mitigate adverse outcomes among mothers and infants under stress right now. As we noted, there are well-known relational interventions that strengthen families systems and stand to buffer the biological cascades of prenatal stress and social isolation on infants. Likewise, mental health interventions (like those to enhance social support and reduce stress) have been integrated successfully into primary, pediatric, and obstetric care in the past, demonstrating positive patient outcomes and reduced 
healthcare costs (e.g., [28]). COVID-19 has complicated the landscape of risks to mothers and infants; in response, we must come together to deliver cost-effective interventions with high potential to mitigate these risks.

\section{Summary}

This manuscript suggests that the COVID-19 pandemic threatens family systems and family health through the compounded effects of stress and social isolation on mothers during the pre- and post-natal periods. We suggest that these risk factors may have toxic inflammatory effects on the bodies of both mothers and infants, with implications for mental health in both individuals as well as long-term developmental consequences for infants. Recommendations for novel research, policy changes, and integrated clinical care build upon the available science.

\section{Funding None.}

\section{Compliance with Ethical Standards}

Conflict of interest The authors declare no conflicts of interest.

\section{References}

1. Belsky J (1984) The determinants of parenting: a process model. Child Dev 55(1):83-96

2. Burchinal MR, Follmer A, Bryant DM (1996) The relations of maternal social support and family structure with maternal responsiveness and child outcomes among African American families. Dev Psychol 32(6):1073-1083. https://doi. org/10.1037/0012-1649.32.6.1073

3. Green BL, Furrer C, McAllister C (2007) How do relationships support parenting? Effects of attachment style and social support on parenting behavior in an at-risk population. Am J Commun Psychol 40(1-2):96-108. https://doi.org/10.1007/s 1046 4-007-9127-y

4. Barnett MA, Mortensen JA, Tilley EH, Gonzalez H (2013) Global and parenting-specific social support as protective factors for the well-being of Mexican American mothers of toddlers. Fam Sci 4(1):98-109. https://doi.org/10.1080/19424620.2013.807294

5. Casagrande AP, Loureiro SR (2014) Analysis of studies on social support and children of depressed mothers: a systematic review. Paideía 24(59):397-405. https://doi.org/10.1590/1982-43272 459201414

6. Slykerman RF, Thompson JMD, Pryor JE, Becroft DMO, Robinson E, Clark PM et al (2005) Maternal stress, social support and preschool children's intelligence. Early Hum Dev 81(10):815821. https://doi.org/10.1016/j.earlhumdev.2005.05.005

7. Laplante DP, Brunet A, Schmitz N, Ciampi A, King S (2008) Project Ice Storm: prenatal maternal stress affects cognitive and linguistic functioning in 5 1/2-year-old children. J Am Acad Child Adolesc Psychiatry 47(9):1063-1072. https://doi.org/10.1097/ CHI.0b013e31817eec80
8. Zhang W, Rajendran K, Ham J, Finik J, Buthmann J, Davey K, Pehme PM, Dana K, Pritchett A, Laws H, Nomura Y (2018) Prenatal exposure to disaster-related traumatic stress and developmental trajectories of temperament in early childhood: superstorm Sandy pregnancy study. J Affect Disord 234:335-345. https://doi. org/10.1016/j.jad.2018.02.067

9. King S, Dancause K, Turcotte-Tremblay AM, Veru F, Laplante DP (2012) Using natural disasters to study the effects of prenatal maternal stress on child health and development. Birth Defects Res Part C 96(4):273-288

10. Veru F, Dancause K, Laplante DP, King S, Luheshi G (2015) Prenatal maternal stress predicts reductions in CD4+ lymphocytes, increases in innate-derived cytokines, and a Th2 shift in adolescents: project ice storm. Physiol Behav 144:137-145

11. Jiang NM, Cowan M, Moonah SN, Petri WA Jr (2018) The impact of systemic inflammation on neurodevelopment. Trends Mol Med 24(9):794-804

12. O'Shea TM, Joseph RM, Kuban KC, Allred EN, Ware J, Coster T et al (2014) Elevated blood levels of inflammation-related proteins are associated with an attention problem at age 24 mo in extremely preterm infants. Pediatr Res 75(6):781-787

13. Leviton A, Allred EN, Fichorova RN, Kuban KC, Oshea TM, Dammann O, ELGAN Study Investigators (2016) Systemic inflammation on postnatal days 21 and 28 and indicators of brain dysfunction 2 years later among children born before the 28th week of gestation. Early Human Dev 93:25-32

14. Kuban KC, Joseph RM, O'Shea TM et al (2017) Circulating inflammatory-associated proteins in the first month of life and cognitive impairment at age 10 years in children born extremely preterm. J Pediatr 180:116-123

15. Lee SE, West KP Jr, Cole RN, Schulze KJ et al (2016) General intelligence is associated with subclinical inflammation in Nepalese children: a population-based plasma proteomics study. Brain Behav Immun 56:253-263

16. Junttila IS (2018) Tuning the cytokine responses: an update on interleukin (IL)-4 and IL-13 receptor complexes. Frontiers Immunol 9:888

17. Costanzo ES, Lutgendorf SK, Sood AK, Anderson B, Sorosky J, Lubaroff DM (2005) Psychosocial factors and interleukin-6 among women with advanced ovarian cancer. Cancer 104(2):305-313

18. Lutgendorf SK, Anderson B, Sorosky JI, Buller RE, Lubaroff DM (2000) Interleukin-6 and use of social support in gynecologic cancer patients. Int J Behav Med 7(2):127-142

19. Fagundes CP, Bennett JM, Derry HM, Kiecolt-Glaser JK (2011) Relationships and inflammation across the lifespan: social developmental pathways to disease. Soc Pers Psychol Compass 5(11):891-903

20. Nelson BW, Bernstein R, Allen NB, Laurent HK (2019) The quality of early infant-caregiver relational attachment and longitudinal changes in infant inflammation across 6 months. Dev Psychobiol 62:674-683

21. Berlin LJ, Zeanah CH, Lieberman AF (2016) Prevention and intervention programs to support early attachment security: a move to the level of the community. Handb Attach Theory Res Clin Appl 3:739-758

22. Dantzer R, O’Connor JC, Freund GG, Johnson RW, Kelley KW (2008) From inflammation to sickness and depression: when the immune system subjugates the brain. Nat Rev Neurosci 9(1):46-56

23. de Araújo LA, Veloso CF, de Campos Souza M, de Azevedo JMC, Tarro G (2020) The potential impact of the COVID-19 pandemic on child growth and development: a systematic review. J Pediatr. https://doi.org/10.1016/j.jped.2020.08.008

24. Davenport MH, Meyer S, Meah VL, Strynadka MC, Khurana R (2020) Moms are not ok: COVID-19 and maternal mental health. Frontiers Glob Women Health 1:1 
25. Center for Translational Neuroscience (2020) How long can the Levee hold? The role of caregiver emotional support in buffering children from the stress of material hardship. Rapid Assessment of Pandemic Impact on Development Early Childhood Survey. https://medium.com/rapid-ec-project/how-long-can-the-levee -hold-2a2cd0779914

26. Dominguez TP, Dunkel-Schetter C, Glynn LM, Hobel C, Sandman CA (2008) Racial differences in birth outcomes: the role of general, pregnancy, and racism stress. Health Psychol 27(2):194

27. Christian LM, Glaser R, Porter K, Iams JD (2013) Stress-induced inflammatory responses in women: effects of race and pregnancy. Psychosom Med 75(7):658
28. Coventry PA, Hudson JL, Kontopantelis E, Archer J et al (2014) Characteristics of effective collaborative care for treatment of depression: a systematic review and meta-regression of 74 randomised controlled trials. PLoS ONE 9(9):e108114

Publisher's Note Springer Nature remains neutral with regard to jurisdictional claims in published maps and institutional affiliations. 